\title{
Design of RLS Adaptive Filter Equivalent for Human Body Communication Channel
}

\author{
Rashmi Baweja \\ Principal Investigator \\ Dept. of Electronics Engg. \\ Raj. Tech. Univ., Kota-324010, \\ Rajasthan, India
}

\author{
Rajeev Gupta \\ Prof. \& Mentor Incharge \\ Dept. of Electronics Engg. \\ Raj. Tech. Univ., Kota-324010, \\ Rajasthan, India
}

\author{
Nishtha Bhagat \\ B.Tech. Student \\ Dept. of Inst. and Control Engg. \\ Netaji Subhas Institute of Tech. \\ Delhi-110078, India
}

\begin{abstract}
Human body can be used as a communication channel for electrical signal transmission and thus offers a novel data communication means in biomedical monitoring systems. Human Body communication channel (on-body) may be proven as promising solution for Wireless Body Area networks (WBANs) in terms of simplicity, reliability, powerefficiency and security. This study proposes the design of an adaptive filter equivalent for human body communication channel. The simulations are based on Electronics and Telecommunication Research Institute (ETRI's) measurement results obtained on human body within a frequency range of $5-50 \mathrm{MHz}$. The measured frequency response is processed to obtain FIR filter matrix coefficients and further identified as RLS adaptive filter. The designing is done using system identification tool in MATLAB. Also a comparison is made between RLS and normalized LMS algorithm for adaptive filter design, which established the RLS adaptive filter as the promising solution for modeling Human Body Communication Channel.
\end{abstract}

\section{General Terms}

Biomedical Electronics

\section{Keywords}

Adaptive Filter, Body Area Network, Human Body Communication, System Identification.

\section{INTRODUCTION}

Human body communication(HBC) also referred as Bodycoupled communication or Intra body communication in literature is a promising solution for Wireless Body Area Networks (WBANs). In this type of communication the human body act as a transmission medium for electrical signals over a frequency range of $1 \mathrm{MHz}-100 \mathrm{MHz}$.

For the frequencies more than $100 \mathrm{MHz}$ human body act like antenna and the communication is no longer limited to human body. Advantage of using human body as transmission media is, full coverage is provided, while at the same time the communication range is limited to the close proximity of the human body. It largely prevents interference between HBC based WBANs and results in frequency reuse factor close to unity i.e. every WBAN can use the same frequency band [1]. Conventional RF and UWB frequencies require complex RF circuitry at higher powers of the order of $\mathrm{mW}$. Whereas, direct transmission of signals through human body requires less power due to absence of high frequency front-ends [1].

Topology of WBAN constitutes two types of nodes, a Central Processing node (CPN) and many sensor nodes to monitor vital signals or signals of interest over the body. The traffic within WBAN is most of the time transmit only from sensor node to CPN node whereas CPN nodes communicates in transmit as well as in receiving mode. CPN sends wake-up signals and signals of critical conditions to the sensor nodes that require low data rates, at the same time must be highly prioritize, secure and consume minimum of the power. This type of communication can suitably be achieved using HBC [2]. Only one node of WBAN i.e. Central Processing Node (CPN) needs to be communicate wirelessly with other devices like, computer, Bluetooth, LAN or internet. This configuration improves battery-life up-to $100 \%$ for sensor nodes and thus adds to the key issue of low power consumption in WBANs. Specifically ECG, Pulse oximetry or body temperature surveillance are key application areas [3]. As reported in many papers, HBC can be achieved via three mechanisms: simple circuit type, capacitive coupling type, and galvanic coupling type [4].

This paper is based on measurement campaign carried out by Electronics and Telecommunication Research Institute (ETRI) [5] in which a simple arrangement of on-body (non-invasive) signal electrodes is taken for transmitting and receiving the data signal, through the human body. Human body is considered as lossy dielectric medium having capacitive component. The frequency response is obtained that constitutes change of amplitude due to loss component of body and change in phase due to capacitive component of body.

Using frequency sampling method a FIR filter for the measured frequency response is designed and the filter matrix coefficients are generated. The resultant FIR filter is considered as intrinsic channel for $\mathrm{HBC}$ and the channel matrix is used to evaluate noisy channel output. An equivalent system for human body FIR filter is designed using system identification tool in matlab.

The outline of this paper is as follows: a description of measurement set-up is given in section 2, intrinsic channel model of human body(on body) is presented in section 3 , section 4 constitutes system identification of human body as adaptive filter, section 5 constitutes simulation results and in section 6, conclusion is drawn and future scope is discussed.

\section{MEASUREMENT SET-UP}

In the HBC, a data signal is transmitted through the body of user, so a data communication can be accomplished wirelessly. To transfer a signal between transmitter and body or receiver and body, the transmitter and the receiver for the HBC have a metal plate signal electrode attached to the body. The signal electrode transfers a signal from the transmitter to the body while transmitting signal, or from the body to the receiver while receiving signal. The data is based on ETRI's measurement campaign for IEEE P802.15 working group for Wireless Personal Area Networks (WPANs) [5]. 
The channel model for $\mathrm{HBC}$ is composed of the frequency response and the noise characteristics as shown in Fig.1(source-ETRI).

Individual users of $\mathrm{HBC}$ have a different frequency response. The frequency response has a uniform deviation range due to different transmission distance (limb lengths) and different composition of tissues of each user.

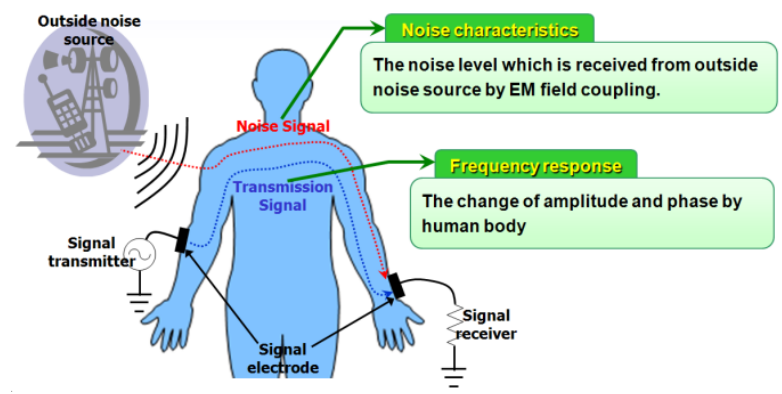

(a)

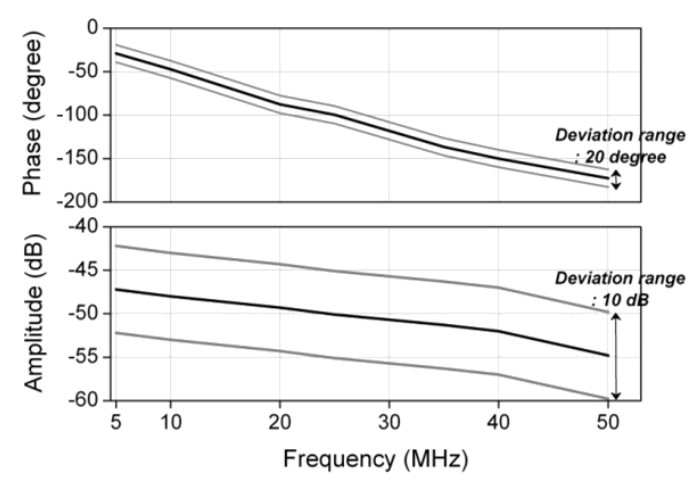

(b)

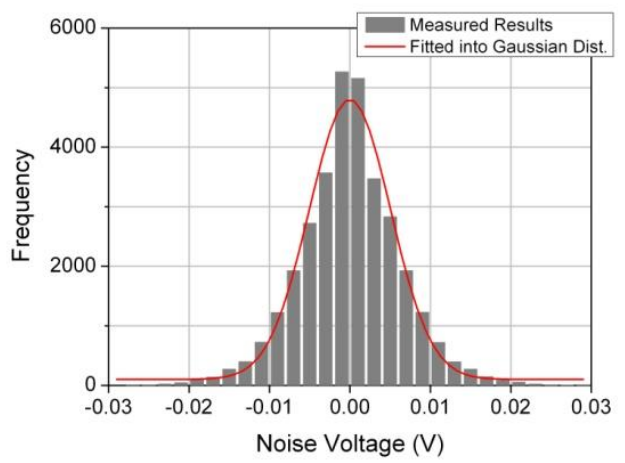

(c)

Figure1[5]. (a) Channel model for HBC, (b) Frequency response and (c) Noise characteristics

External EM waves causes a noise signal inside body, this noise signal as well as the data signal is received at the receiver electrode. The noise voltage is measured with multiple locations for a long time, the site where the largest noise voltage is measured for the longest time is selected and its statistical parameters are defined as the noise characteristics.

The frequency response had been taken in the frequency range of $0-55 \mathrm{MHz}$ with the steps of $5 \mathrm{MHz}$. The values at 0 and 55 $\mathrm{MHz}$ are interpolated The response as shown in Fig.1(b) is obtained by locating the transmitter and receiver electrode on the fingertips of thumb of each hand (at transmission distance of $150 \mathrm{~cm}$ ), the size of metallic signal electrode is $2 \times 2 \mathrm{~cm}^{2}$ and the load impedance of receiver electrode is $10 \mathrm{Mohm}$. The measured noise has a Gaussian distribution as shown in Fig.1(c). The mean and variance values are zero and $2.55 \times 10$ 5 respectively.

\section{FIR FILTER EQUIVALENT FOR HUMAN BODY COMMUNICATION CHANNEL}

The frequency response constitutes channel attenuation in $\mathrm{dBs}$ and phase change in degrees over the frequency range 0-55 MHz. FIR filter channel matrix is generated using frequency sampling technique for filter design. The sampling frequency is taken as $100 \mathrm{MHz}$. Frequency sampling technique is used to design non prototype filters having desired frequency response of any irregular shape with filter's transition bandwidth equal to the transition bandwidth chosen. Interpolated frequency response is same as desired frequency response only at sampled frequencies and there will be finite error present at all other frequencies. Time response is evaluated from the Inverse Fast Fourier transform of the available frequency response.

\section{SYSTEM IDENTIFICATION OF HBC CHANNEL}

One common application of adaptive filters is to identify an unknown system, such as the response of an unknown communications channel[6]. Other applications include echo cancellation and channel identification. In the figure, the unknown system(human body FIR filter) is placed in parallel with the adaptive filter.

Clearly, when $\mathrm{e}(\mathrm{k})$ is very small, the adaptive filter response is close to the response of the human body FIR filter(unknown) system. In this case the same input feeds both the adaptive filter and the unknown.

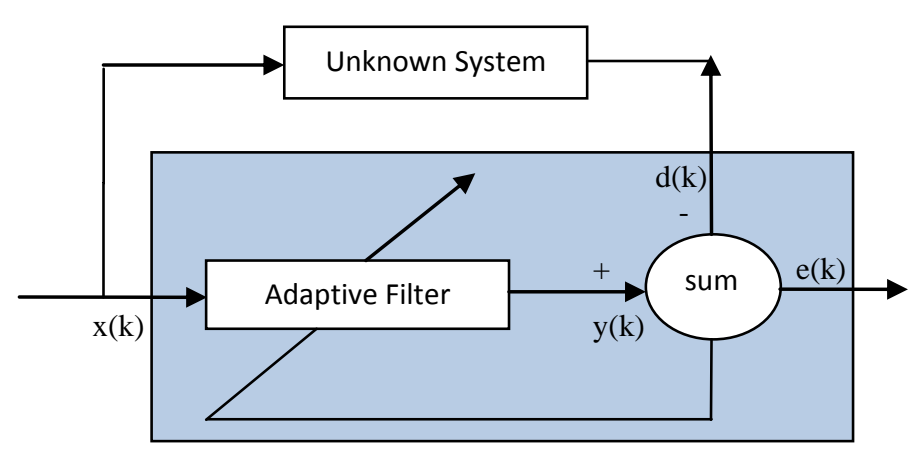

Fig 2[6]: Using an Adaptive Filter to Identify an Unknown System

$\mathrm{X}(\mathrm{k})$ is the input data bits $( \pm 1)$ taken 1000 samples at a time. The desired signal $\mathrm{d}(\mathrm{k})$ is the noisy channel output data or the response of human body system. Error signal $\mathrm{e}(\mathrm{k})$ i.e. difference between desired signal $\{\mathrm{d}(\mathrm{k})\}$ and adaptive filter output $\{\mathrm{y}(\mathrm{k})\}$, is to be minimised.

\subsection{RLMS Algorithm}

The Recursive least squares (RLS) is an adaptive filter which recursively finds the coefficients that minimize a weighted linear least squares cost function relating to the input signals. This is in contrast to other algorithms such as the least mean squares (LMS) that aim to reduce the mean square error. In the derivation of the RLS, the input signals 
are considered deterministic, while for the LMS and similar algorithm they are considered stochastic. Compared to most of its competitors, the RLS exhibits extremely fast convergence. However, this benefit comes at the cost of high computational complexity[7-9].

The Recursive least squares (RLS) adaptive filter is an algorithm which recursively finds the filter coefficients that minimize a weighted linear least squares cost function relating to the input signals[10-11]. The RLS algorithms are known for their excellent performance when working in time varying environments but at the cost of an increased computational complexity and some stability problems. In this algorithm the filter tap weight vector is updated using Eq.

$\mathrm{w}(\mathrm{n})=\overline{\mathrm{w}}^{\mathrm{T}}(\mathrm{n}-1)+\mathrm{k}(\mathrm{n}) \overline{\mathrm{c}}_{\mathrm{n}-1}(\mathrm{n})$

$\mathrm{k}(\mathrm{n})=\mathrm{u}(\mathrm{n}) /\left(\lambda+\mathrm{X}^{\mathrm{T}}(\mathrm{n}) \mathrm{u}(\mathrm{n})\right)$

$\mathrm{u}(\mathrm{n})=\overline{\mathrm{w}}_{\lambda}^{-1}(\mathrm{n}-1) X(\mathrm{n})$

Eq. (2) and (3) are intermediate gain vector used to compute tap weights.

Where $\lambda$ is a small positive constant very close to, but smaller than 1. The filter output is calculated using the filter tap weights of above iteration and the current input vector as in Eq. (4).

$$
\begin{aligned}
& \overline{\mathrm{y}}_{\mathrm{n}-1}(\mathrm{n})=\overline{\mathrm{w}}^{\mathrm{T}}(\mathrm{n}-1) \mathrm{X}(\mathrm{n}) \\
& \overline{\mathrm{e}}_{\mathrm{n}-1}(\mathrm{n})=\mathrm{d}(\mathrm{n})-\overline{\mathrm{y}}_{\mathrm{n}-1}(\mathrm{n})
\end{aligned}
$$

In the RLS Algorithm [12] the estimate of previous samples of output signal, error signal and filter weight is required that leads to higher memory requirements.

\section{SIMULATION RESULTS}

An equivalent FIR filter is designed for HBC channel and the unknown FIR filter response constituting both amplitude and phase change, is identified as adaptive filter using system identification tool in MATLAB. Adaptive RLS filter is designed with filter length 40 and step size 0.99 is designed for 500 iterations as shown in fig.4. The designed RLS is compared with earlier designed nLMS filter with signal value and coefficient as shown in fig. 3[13]. The two structures are analysed and compared. Also a comparison of error signal for 500 iterations is shown in fig.5. It is clear from fig.5 that RLS algorithm take almost half the time required to reach steady state as compared to the nLMS algorithm also the magnitude of steady state error is less and stability is enhanced in case of RLS filter as compared to nLMS filter. Obtained results are in accordance with previous findings as mentioned in table 1[14]. Thus RLS adaptive filter resembles well with the human body communication channel as error convergence rate is faster and better for this system. Also the actual filter coefficients and the estimated coefficient values resembles closely, hence the human body can be identified as RLS adaptive filter of length 40 and step size of 0.99 .
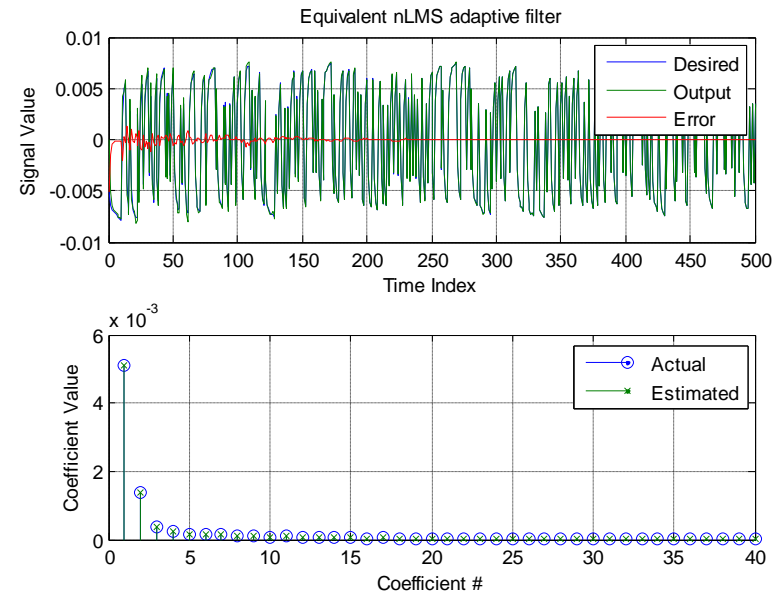

Fig. 3 Equivalent nLMS adaptive filter for 500 iterations[13].
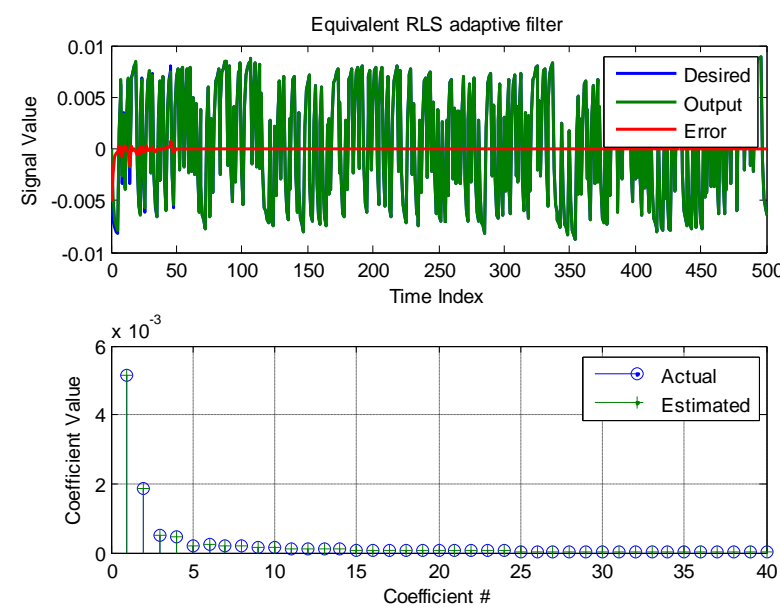

Fig. 4 Equivalent RLS adaptive filter for 500 iterations.

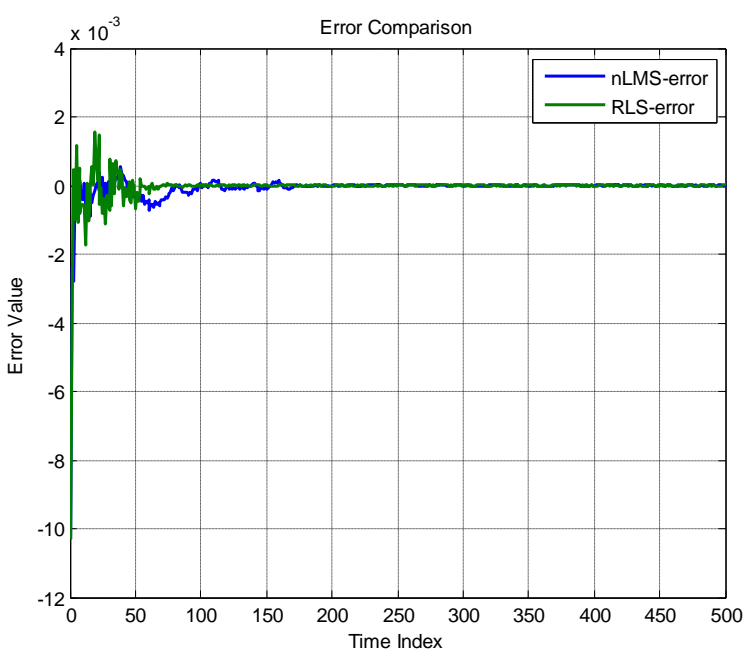

Fig. 5 Comparison of RLS error and nLMS error 
Table 1: Performance Comparison of nLMS and RLS algorithms[14]

\begin{tabular}{|l|l|l|l|l|}
\hline $\begin{array}{l}\text { S. } \\
\text { No. }\end{array}$ & Algorithms & MSE & Complexity & Stability \\
\hline 1. & nLMS & $1.5^{*} 10-2$ & $3 \mathrm{~N}+1$ & Stable \\
\hline 2. & RLS & $6.2 * 10-3$ & $4 \mathrm{~N} 2$ & $\begin{array}{l}\text { Highly } \\
\text { Stable }\end{array}$ \\
\hline
\end{tabular}

\section{CONCLUSION AND FUTURE SCOPE}

Human Body Communication appears to be a better solution for WBAN's in terms of reliability, security and power efficiency. HBC channel has signal attenuation of upto $57.5 \mathrm{~dB}$ for frequency range of $0-55 \mathrm{MHz}$. An equivalent FIR filter is developed for HBC channel using frequency sampling method. The unknown HBC channel(FIR filter) is further identified as an adaptive RLS filter and compared with nLMS adaptive filter designed earlier[13]. The designed RLS filter with filter length 40 and step size 0.99 closely mimics the HBC channel. Error converges well and faster in case of RLS adaptive filter as compared to nLMS, thus a standing human body can be considered as an RLS adaptive filter. The proposed equivalent can be used for further simulations to establish human body as a communication channel. In future the effect of chosen frequency range on the human body needs to be analysed. Also the influence of body movement in terms of noise over human body channel may also be considered.

\section{ACKNOWLEDGMENTS}

This work is a part of research project financially sponsored by Department of Science and Technology, New Delhi, under Women Scientist Scheme (WOS-A) with ref. no.SR/WOSA/ET-116/2012. The research is carried out at Department of Electronics Engineering, Rajasthan Technical University, Kota, Rajasthan, India.

\section{REFERENCES}

[1] Tim C.W. Schenk, Nafiseh Seyed Mazloum, Luc Tan, Peter Rutten, Experimental Characterization of the BodyCoupled Communication Channel, IEEE ISWCS 2008.

[2] Arthur Astrin, Measurements of body channel at 13.5 MHz, IEEE 802.15-08-0590-00-0006, August 2008.

[3] Marc Simon Wegmueller, Michael Oberle, An Attempt to Model the Human Body as a Communication Channel,
IEEE Transactions on Biomedical Engineering, Vol. 54, No.10, October 2007

[4] Xinzhuo Liu, Xianqing Yang, Yuan Wang, and Lei Wang, Comprehensive Measurements on Body Channel Characteristics of Human Body Communication, ICMMT 2010 Proceedings.

[5] Jung-Hwan Hwang, Il-Hyoung Park, and Sung-Weon Kang, Channel model for human body communication, IEEE 802.15-08-0577-00-0006, August 2008.

[6] Filter Design Toolbox (C) 1984-2007 The MathWorks, Inc.

[7] John G. Proakis, "Digital Signal Processing Principles, Algorithms and Applications", Pearson Prentice Hall, fourth Edition, page No. 909-911.

[8] Simon Haykin: Adaptive Filter Theory, Prentice Hall, 2002, ISBN 0-13-048434-2.

[9] Simon S. Haykin, Bernard Widrow (Editor): LeastMean-Square Adaptive Filters, Wiley, 2003, ISBN 0471-21570-8.

[10] Monson H. Hayes: Statistical Digital Signal Processing and Modeling, Wiley, 1996, ISBN 0471-59431-8.

[11] Paulo S.R. Diniz: Adaptive Filtering: Algorithms and Practical Implementation, Kluwer Academic Publishers, 1997, ISBN 0-7923-9912-9.

[12] Raj Kumar Thenua and S.K. Agarwal "Simulation and Performance Analyasis of Adaptive Filter in Noise Cancellation" International Journal of Engineering Science and Technology Vol. 2(9), 2010, 4373-4378.

[13] Rashmi Baweja, Rajeev Gupta and Neeraj Bhagat, "A Comparison of LMS and nLMS Adaptive Filter Equivalent for Human Body Communication Channel", Proceedings of IRF International Conference, $22^{\text {nd }}$ March-2015, Jaipur, India, 16-20, ISBN: 978-93-8270280-1.

[14] Jyoti Dhimani, Shadab Ahmed and Kuldeep Gulia, Comparison between Adaptive filter Algorithms (LMS, NLMS and RLS) International Journal of Science, Engineering and Technology Research (IJSETR) Vol. 2(5), May 2013, pp 1100-03. 